Pacific Journal of Mathematics

RELATIVE WIDTH MEASURES AND THE PLANK PROBLEM 


\title{
RELATIVE WIDTH MEASURES AND THE PLANK PROBLEM
}

\section{R. J. GARDNER}

\begin{abstract}
A relative width measure in a convex body $K$ in $R^{\prime \prime}$ for a set 6 of directions is a Borel probability measure in $K$ such that the measure of the intersection of $K$ with each slab orthogonal to a direction in 6 is equal to the relative width of the slab. Such measures are studied in connection with the unsolved plank problem of Th. Bang.
\end{abstract}

0. Introduction. Tarski's plank problem was solved by Th. Bang [2] when he showed that if a convex body $K$ in $\mathrm{R}^{\prime \prime}$ is covered by a finite number of slabs, the sum of their widths is at least the minimum width of $K$. Bang conjectured that a stronger, and affine invariant, inequality should hold; namely, that the sum of the relative widths of the slabs is at least one (the relative width of a slab is its width divided by the width of $K$ in the same direction). This is still unsolved.

A relative width measure is a Borel probability measure in $K$ such that the measure of the intersection of $K$ with any slab is precisely the relative width of the slab. An example, known to Achimedes, is nor-

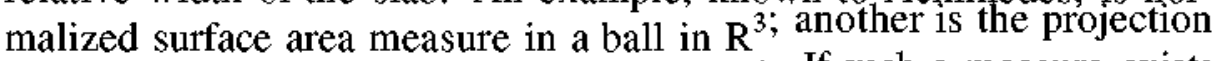
of this measure, normalized, in a disc in $\mathrm{R}^{2}$. If such a measure exists in $K$, then Bang's conjecture is true for $K$. This observation has been made several times in the literature, but does not seem to have been thoroughly investigated.

We study these measures, always with Bang's conjecture in mind. For this application, the measures need only have the relative width property for directions corresponding to the covering slabs, and in fact a reduction shows that we need only seek them for coordinate directions. Theorem 1 shows that measures with the latter property always exist in $\mathrm{R}^{2}$, which generalizes the known special case of Bang's conjecture for two slabs. However, Example 2 shows that even measures with this weaker property do not generally exist for $K$ in $\mathrm{R}^{3}$.

Section 3 concerns measures with the relative width property for infinite sets of directions. Here, using Fourier transform techniques and particularly a method due to $\mathrm{K}$. Falconer, we show (Theorem 3) that measures with the relative width property for all directions do 
not exist in the ball in $\mathrm{R}^{\prime \prime}$ for $n>3$. (After this paper was written, I learned that $G$. Schwarz also proves this in [23].) In Theorem 4 we refine this result, and show that those in the disc in $\mathrm{R}^{2}$ and ball in $\mathrm{R}^{3}$ are essentially the only such measures. Sufficiently 'large' infinite sets

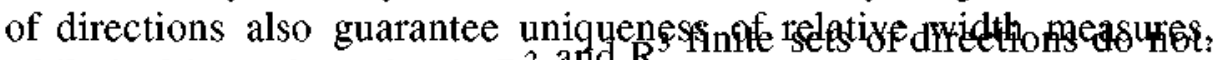
while in $\$ 4$ we show that in $\mathrm{R}^{2}$ and $\mathrm{R}$

I thank Don Chakerian for many stimulating and helpful discussions on Bang's plank problem, and for providing some useful references; and L. Zalcman for supplying the case $n=3$ of Theorem 5.

1. Relative width measures and the plank problem. We shall write int $E$ and $d E$ for the interior and boundary of a set $E$, respectively.

Suppose $K$ is a compact convex set in $\mathrm{R}^{\prime \prime}$, and 6 is a direction (which we identify throughout with the corresponding unit vector in $\mathrm{R}^{\prime \prime)}$. We denote by $W(K, 6)$ the width of $K$ in the direction 0 ; that is, the distance between two hyperplanes which are orthogonal to 8 and which support $K$. If AT is a fixed compact convex set, and $H$ is a convex set, the relative width of $H$ in the direction 6 , when $W(K, 6) / 0$, is

$$
w(H, 6)=W(H \cap K, 6) / W(K, 6) .
$$

A slab orthogonal to 6 is the closed set between two hyperplanes which are orthogonal to 6 .

A measure is a non-negative set function, assumed countably additive unless otherwise stated. Let $/ z$ be a Borel probability measure in the compact convex set $K$, and let 0 be a set of directions. We say that $n$ is a relative width measure in $K$ for $\mathrm{G}$ if

$$
\mu(S \cap K)=w(S, \theta)
$$

whenever $S$ is a slab orthogonal to some $6 \mathrm{G} \mathrm{G}$.

Suppose now that $\mathrm{G}=\left\{0, \ldots, \sigma_{k}\right\}$ is a finite set of directions, $K$ is a compact convex set in $\mathrm{R}^{\prime \prime}$ and $5^{\prime \prime}$; is a slab orthogonal to $0_{i}, 1 \leq / \leq k$. If $K \subset V_{i S i}$, is it true that $J 2 i W\left(S_{i, 6 i}>-1\right.$ ? This is Bang's plank problem (see [3]). It is an affine invariant form of Tarski's plank problem [24], solved by Bang in [2] when he showed that the weaker inequality

$$
\sum_{i} W\left(S_{i} \cap K, \theta_{i}\right) \geq \min _{\theta}\{W(K, \theta)\}
$$

holds.

It is not a new observation that relative width measures are relevant to Bang's plank problem. This has essentially been noted by G. Hajós 
and A. Rényi [20], D. Ohmann [17] and J. W. Green [12], among others. For, if $n$ is a relative width measure in $K$ for $0=\left\{6, \ldots, \theta_{k}\right\}$, then

$$
\underset{i}{\mathfrak{f}}>(\mathbf{S}, \mathbf{D K}) \geq \mu(\boldsymbol{K})=1 \Rightarrow \sum_{i} w\left(S_{i}, \theta_{i}\right) \geq 1,
$$

as required.

Since there seems to be some confusion about the status of Bang's problem, we shall briefly survey attempts to solve it. In [17], D. Ohmann shows that it suffices to consider a convex body $K$ in R" covered by $n$ slabs $S, \ldots, S_{n}$, with 5 , 'orthogonal to the $/$ th coordinate axis. Further, a signed relative width measure is constructed in $K$ for these directions, but this is not enough for the implication (2), even when $f i(S j \mathrm{n} K)>0$ for each /. These and other remarks on the problem may be found in [4], where it is also shown that Bang's conjecture is true for covers of $K$ by two slabs. Other proofs of this special case are given in [1] and [15].

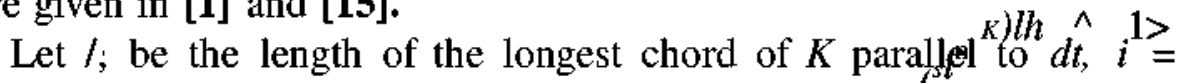
$\nmid \ldots, k$. Then Bang's proof actually shows that $Y . t$

which is stronger than (1). According to a translation, the paper [14] in Chinese only confirms this result, despite its title. Another attempt in [18] breaks down; the error is pinpointed in the review by $\mathrm{C}$. A. Rogers cited under [18].

Lastly, we note variations in the proof of Bang's result ([6], [10], special cases in [7], [9]) and the interesting paper of R. Alexander [1] which relates Bang's problem for $K$ a square to an unsolved problem of Davenport.

We begin by considering those properties of $p i$ necessary to derive (2). We actually only need $j u$ to be finitely additive and to satisfy $f i(S \cap K) \leq w(S, 6)$ for each slab $S$ orthogonal to 6 . Assuming only this, we note that the support of // must lie in $K$ for a straightforward application to the plank problem. For if $K \mathrm{c} \mathrm{R}^{2}, 0$ contains at least two directions, and $l\left(\mathrm{R}^{2}-K\right)>0$, it is easy to find slabs $S i$ and $\wedge 2$ orthogonal to any two of these directions such that $K \subset S V) S 2$ and $f(S \backslash \mathrm{U} S 2)=c<1$, from which we can only deduce that $c \leq$ $w(S \backslash 61)+w(S 2,62)$. Now the first two lemmas show that we lose no generality in making our other stronger assumptions on $f i$.

LEMMA 1. Suppose fi is a finitely additive Borel probability measure in $K$, such that fi( $S \cap K) \leq w(S, 6)$ for all slabs $S$ orthogonal to 6 . Then $n(S D K)=w(S, 6)$ for all such slabs. 
Proof. Let $S \backslash$ be any slab orthogonal to 6 . We can find two disjoint slabs $£ 2$ and $\mathrm{S} 3$, both orthogonal to 6 , with $K \subset V_{t i S i}$ and $52 i W(S i, O)=l$. Then

$$
\left.1 \leq \underset{i}{ } /^{*}(\$ \mathrm{n} K) \leq n / S_{x} C K\right)+w\left(S_{2}, d\right)+w\left(S_{3}, 6\right),
$$

so $f i(S i n K)>l-w\left(S_{2}, d\right)-w\left(S_{2}, 6\right)=w(S !, d)$, giving $/ i($ Si $f K)=$ $w(S i, 6)$ as required.

LEMMA 2. Suppose $n$ is a finitely additive probability measure in $K$, defined on the algebra si generated by sets $S D K$, where $S$ is a slab orthogonal to $d$, and 6 belongs to a fixed set 0 ofdirections. Suppose also that fi $(S \mathrm{n} K)=w(S, 6)$ for such sets. Then $f i$ can be extended to a relative width measure in $K$ for $\mathrm{G}$.

Proof. Let $A$ e siff. Then $A=\mathrm{U}^{m}=\mathrm{i} A j$, where for each $i$ there is a polytope $P i$ such that

$$
\text { (int } \left.P_{i}\right) \cap K \subset A_{i} \subset P_{i} \cap K,
$$

and moreover for each face of $P i$ there is a $6 \mathrm{e} C$ such that this face is orthogonal to 6 .

For each $/$, let $H i$ be a finite union of open slabs, each orthogonal to some $6 € 0$, such that

$$
P i-i n t P_{t} \quad a \quad H i
$$

and the sum of the relative widths of the slabs in $H i$ is less than $\mathrm{e} / \mathrm{m}$.

Let $Q=(P i-H i) D K$ for each $/$, and $C=(\mathrm{J} £ \mathrm{i} Q$. Then $C$ is compact, $C \subset A$, and

$$
\mu(C) \geq \mu\left(\bigcup_{i} P_{i} \cap K\right)-\mu\left(\bigcup_{i} H_{i} \cap K\right)>\mu(A)-\varepsilon,
$$

where we have used the fact that $\mathrm{P}, \mathrm{n} K, H_{t} \mathrm{n} K$ and $\mathrm{C}$ all belong to $\mathrm{si}$.

This shows that $f i$ is inner regular on $\mathrm{J} /$ with respect to the compact sets. By Henry's extension theorem [22, p. 51, Theorem 16], $f i$ can be extended to a countably additive Borel measure.

REMARKS, (i) The extension provided by Henry's theorem is unique if the algebra si contains a base for the topology of $\mathrm{R}$ ". This will be the case if 0 contains $n$ linearly independent directions. However, 
even if $s f$ does contain a base, there may be more than one relative width measure in $K$ for 0 (see Theorem 5 ).

(ii) Given any set $\mathcal{C}$ of directions, we may of course define $f i$ on the sets $S$ n $K$ of Lemma 2 by $f i(S \cap K)=w(S, 6)$. However, $/ i$ may not extend to the algebra $s f$, Example 1 shows this.

2. Existence of relative width measures. Here and throughout $X$ denotes linear Lebesgue measure in $\mathrm{R}^{\prime \prime}$.

Suppose $K$ is a convex body in $\mathrm{R}^{\prime \prime}$ and 6 is a direction. Let / be a chord of $K$ meeting the two supporting hyperplanes to $K$ which are orthogonal to 6 . Define $/ i$ in $K$ by $/ i(B)=X / B n /) / / !(/)$. Clearly $n$ is a relative width measure in $K$ for $\{\#\}$.

If $n$ is a relative width measure in $K$ for (C), and $4>$ is a nonsingular affine transformation, there is a corresponding relative width measure in $c j)(K)$. Define $/ \mathrm{z}^{\wedge \prime 1}$ on $\left(^{(1)}\right.$ by

$$
\left(\mu \phi^{-1}\right)(B)=\mu\left(\phi^{-1}(B)\right)
$$

for each Borel set $B$ in ${ }^{\wedge}\left(\mathrm{A}^{\prime \prime}\right)$. If $6 \mathrm{e} \subset \mathrm{C}$, and /f is a hyperplane orthogonal to 6 , then $0(/ /)$ is a hyperplane orthogonal to some direction $6^{\prime}$. If $8^{\prime}=\left\{\wedge^{\prime}: 0 \mathrm{e}\right.$ (C) $\}$, it is easy to see that $\left.n<j\right)^{l}$ is a relative width measure in $\langle f)(K)$ for ${ }^{\circ}$.

THEOREM 1. Let Kbea convex body in $\mathrm{R}^{\prime \prime}$ and 0 6i two directions. Then there is a relative width measure in $K$ for $\{61,62\}$.

Proof. If $n>2$, let $P$ be the span of the directions 6 and 92 , and let *F denote projection onto $P$. Let $E$ be any Borel subset of $K$ for which * $\neq$ is a bijection from $E$ to $* \mathrm{~F}(\mathrm{X})$ (such a set exists; see [16, 4D.13]). If $/ /$ is a relative width measure in *F(AT) for $\{0 j, 02\} »$ define $/ \mathrm{i}$ in $\mathrm{A}$ by

$$
\tilde{\mu}(B)=\mu(\Psi(B \cap E))
$$

for Borel sets $B C K$. Then $/ \mathrm{i}$ is a relative width measure in $K$ for $\{\# 1, \# 2\}$ - So it suffices to consider the case $n-2$.

By using an affine transformation, and the remarks preceding this theorem, we may assume that $\delta$ and 62 are parallel to the coordinate axes, and $K$ is contained in the unit square / and meets all its sides. Let a $1, \mathcal{Z}$ be the $\mathrm{x}$-coordinates of any two points in the intersections of $d K$ with the bottom and top sides of $/$, respectively, and $b \backslash b_{2}$ the $y$-coordinates of any two points in the intersections of $d K$ with the right and left sides of $/$, respectively. The four points so obtained 
form the vertices of a quadrilateral $Q \subset K$ (which may degenerate to a triangle).

We consider two cases.

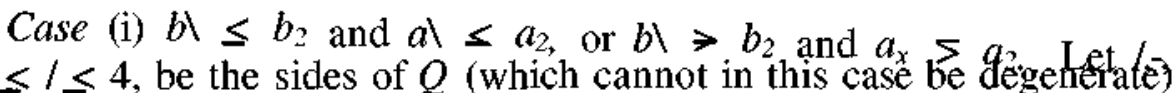
labelled clockwise with $\Lambda$ the segment joining the points $(a \backslash 0)$ and $(0,62)$. Let $m$ be the line segment joining the points $(a \backslash b \backslash)$ and $\left(a_{2}, b_{2}\right)$. For Borel sets $B$ in $K$ define

$$
\begin{aligned}
H(B)= & {[\mathrm{f} 1, \mathrm{M}(5 \mathrm{n} h) / k(h)]+\left[a_{2}\left(l-b_{2}\right) k\left\{B \mathrm{n} l_{2}\right) / \lambda\left(l_{2}\right)\right] } \\
& +\left[\left(1-a_{2}\right)\left(1-b_{1}\right) \lambda\left(B \cap l_{3}\right) / \lambda\left(l_{3}\right)\right] \\
& +\left[\left(1-a_{1}\right) b_{1} \lambda\left(B \cap l_{4}\right) / \lambda\left(l_{4}\right)\right] \\
& +\left[\left(a_{2}-a_{1}\right)\left(b_{2}-b_{1}\right) \lambda(B \cap m) / \lambda(m)\right] .
\end{aligned}
$$

Of course, // is the sum of suitable multiples of $k$ restricted to the line segments /; and $m$. To check that $J U$ is a relative width measure in $K$ for the coordinate directions is now simply a matter of computation.

Case (ii) $b \backslash \geq b_{2}$ and $a_{2}>a \backslash$ (or $b \backslash<b_{2}$ and $a_{2} \geq a$. Let $b$.
$\leq / \leq 4$, be the sides of $Q$ as in Case (i). Since $m$ may not be contained in $Q$, and even if it is, $n$ as defined above does not work, we use instead the two diagonals $d$ (joining $(a \backslash 0)$ and $\left(a_{2,1}\right)$ ) and $d_{2}$ (joining $(0,62)$ and $(, b \cup)$.

We seek non-negative multiples $\mathrm{a}$, and $/ ?_{7}$ of $k$ normalized on $/$; and $d j$, respectively, such that the sum of these measures is the required relative width measure.

Assuming $b \backslash \geq b_{2}$ and $a_{2} \rightarrow a \backslash$ and considering slabs of the form $((x, y): 0 \leq x \leq c, c \leq a)$ we see that the equation

$$
\frac{\alpha_{1}}{a_{1}}+\frac{\alpha_{2}}{a_{2}}+\beta_{2}=1
$$

must be satisfied. Slabs of the form $\left((x, y): a \leq x \leq c, c \leq a_{2}\right)$ and $\left\{(x, y): a_{2}<x<c, c<1\right\}$ yield similar equations, and we obtain three more by looking at horizontal slabs. In addition, we require

$$
\sum \alpha_{i}+\sum \beta_{j}=1
$$

for a probability measure. 
The equations can be solved by setting $f t_{x}=0$ or $p_{2}=0$. If $f i_{x}=0$, we obtain:

$$
\begin{aligned}
& a_{x}=a_{1} b_{1} b_{2} / \Delta_{1}, \\
& a_{2}=a_{2}\left(l-b_{x}\right)\left(l-b_{2}\right) / A_{2} \\
& a_{3}=\quad\left(l-a_{2}\right)\left(l-b_{x}\right)\left(-b_{2}\right) / A_{2}, \\
& 014=\left(1-a_{x}\right) b_{x} b_{2} / A_{x} \text { and } \\
& \left.p_{2}=\left(b_{x} \quad-b_{2}\right)\left(a_{x} b_{x} \cap-b_{2}\right)-a_{2} b_{2} \wedge-b_{x}\right)-a_{x} a_{2}(b
\end{aligned}
$$

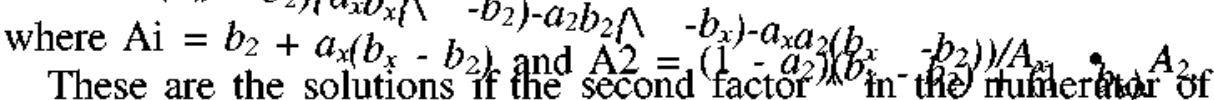
$m$ is non-negative. If not, we set $p_{2}=0$, obtaining solutions for a, from those above by interchanging $a_{x}$ and $b_{2}, a_{2}$ and $b_{x}$, and $a_{2}$ and «4. We also get factor in the numerator of $p_{x}$ by $T_{x}$, we see that $T_{x}+T_{2}=0$, so that $p_{x}>0$ if $r_{2}<0$.

If $a_{x}=a_{2}$ and $b_{x}=b_{2}$ we solve as in Case (i) Degenerate cases where $Q$ reduces to a triangle may be solved as above, and here the measure is supported by the boundary of the triangle.

It follows that Bang's conjecture is true for two slabs, and so Theorem 1 can be regarded as a generalization of this known result. In fact, only Case (i) of Theorem 1 is needed for this. To see this, suppose $K c S_{x} \cup S_{2}$, where $S_{x}$ and $S_{2}$ are slabs orthogonal to the coordinate axes. Let $R=S_{x} \mathrm{n} S_{2}$, and let $Q$ be the quadrilateral obtained by drawing tangent lines to the convex hull of $K$ and $R$ at the vertices of $R$. Now $Q$ c $S_{x} \mathrm{U} \mathrm{S}_{2}$, and we may assume $Q$ is inscribed in the unit square. Then $Q$ is as in Case (i) of Theorem 1 . Since $Q$ is wider than $K$ in the coordinate directions, the existence of a relative width measure in $Q$ gives the result for $K$.

In fact, the measure from Case (i) of Theorem 1 was found by analyzing the proof of Bang's conjecture for two slabs in [15].

In view of the difficulty of finding a relative width measure in $K$ for two directions, it is surprising that there are convex bodies which

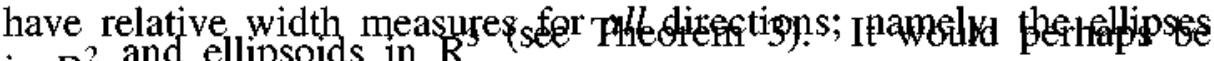
in $\mathrm{R}^{2}$ and ellipsoids in $\mathrm{R}$

unreasonable to expect all convex bodies to support such measures, but the point is that by Ohmann's reduction of Bang's problem, we 
only require a relative width measure in a convex body $K$ in $\mathrm{R}$ " for the $n$ coordinate directions. However, we show in Example 2 that this may not exist, even when $n=3$.

The proof of the following lemma is a slightly modified version of ([8], Theorem 3), due to K. Falconer, which deals only with absolutely continuous $/ /$.

LEMMA 3. Suppose $K$ is a compact convex set in $\mathrm{R}^{\prime \prime}, \mathrm{O}$ is a set of directions, andJU is a relative width measure in Kfor $\odot$. Let the centroid of $K$ with respect to the density dfi be at the origin, and the support function of $K$ be $k(d)$. Then there is a second-degree homogeneous polynomial $p(6)$ such that $k^{2(6)}=p(6)$ for all $6 € 0$.

Proof. Let $6 € \subseteq$, let $L$ be the line through the origin in the direction 8 , and suppose that $v$ is the projection of $f i$ on $L$. Since $/ /$ is a relative width measure in $K$ for $\{6\}$, we have

$$
\nu=(k(\theta)+k(-\theta))^{-1} \lambda_{L},
$$

where $X i$ is A restricted to $L$. Now if / is a 'ridge function'-a function on $K$ which depends only on $x \cdot 6=t$ for all $x \mathrm{e} K$-then

$$
\begin{aligned}
/_{J K} f(x) d f i(x) & =/_{J-l k(-0)} f(t) d u(t) \\
& =(k(\theta)+k(-\theta))^{-1} \int_{J^{k}(-6)}^{k(6)} f(t) d t .
\end{aligned}
$$

Since the centroid of $K$ with respect to $d f i$ is at the origin, we have

$$
\begin{aligned}
0 & \left.=\int_{K} x d j u(x)\right] \quad-6=\jmath_{K} f(x-0) d / i(x) \\
& =\left(k(6)+\left.k(-6) r^{x}\right|_{J-k(-6)} t d t,\right.
\end{aligned}
$$

giving $k(6)=k(-6)$.

Taking second moments, we get

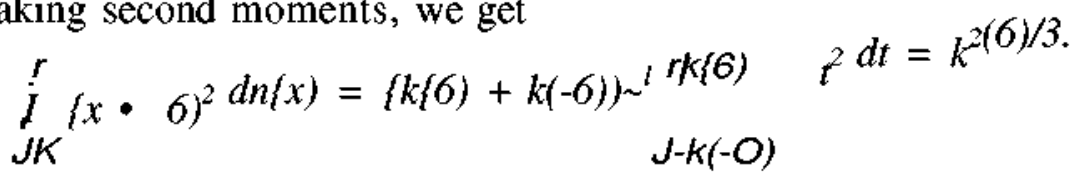

The left-hand side is a second-degree homogeneous polynomial in $6=\left(6, \ldots, \sigma_{n}\right)$, giving the result.

EXAMPLE 1. A convex body $T$ in $\mathrm{R}^{2}$ with no relative width measure for a certain set of three directions. 
Let $T$ be the triangle with vertices at the origin, $(0,1)$ and $(1,0)$, and 9 the set of directions orthogonal to the edges of $T$. Suppose $j u$ were a relative width measure in $T$ for 0 , and let $c$ be the centroid of $T$ relative to $d f i$. From Lemma 3 we require only the fact that if $t(6)$ is the support function of $T$ with respect to $c$, then $t(8)=t(-6)$ for $0 € 0$. From the coordinate directions, we get $c=j j, j$ ), which contradicts $t(6)=t(-d)$ for 6 orthogonal to the hypoteneuse of $T$.

EXAMPLE 2 . A convex body $U$ in $\mathrm{R}^{3}$ with no relative width measure for the three coordinate directions.

Let $U$ be the tetrahedron in $\mathrm{R}^{3}$ with vertices at the origin, $(1,0,0)$, $(0,1,0)$ and $(0,0,1)$. If a relative width measure $/ i$ with centroid $c$ existed, then, as in Example 1, using the $x$ - and y-directions, $c$ N 、a) for some $a>0$. Since $c$ e $U, a=0$, contradicting symmetry of the support function at $c$ in the z-direction.

Many examples such as those above could be obtained in the same way. Let us note, however, that there are convex bodies in $\mathrm{R}^{3}$ without relative width measures for the coordinate directions, to which Lemma 3 cannot be applied. One such is the regular octahedron centered at the origin, with axes in the coordinate directions. This has all the required symmetry, and another proof is needed to show that there is no measure; we omit this here. Despite the non-existence of a relative width measure for this regular octahedron or for the tetrahedron in Example 2, Bang's conjecture is true for 3 slabs covering these polyhedra orthogonal to the coordinate directions. To see this, apply Bang's theorem in its stronger form mentioned in $\S 1$.

3. Existence and uniqueness for infinite 0 . In this section we apply Fourier transform techniques to study the existence and uniqueness of relative width measures in convex bodies for infinite sets of directions.

THEOREM 2. Let Kbea compact convex set in $\mathrm{R} "$, and 0 a (necessarily infinite) set ofdirections with the property that each analyticfunction

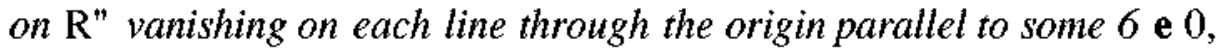
is identically zero. Then ifft is a relative width measure in $K$ for $\odot$, $f i$ is unique.

Proof. Suppose $/ /, I=1,2$, are relative width measures in $K$ for 0 . Let $f i j$ be the Fourier transform of $f i j$. Then for $</ \mathrm{eR}$ " with $d \wedge d=6 € 0$ and $x \cdot 6=t$, we have

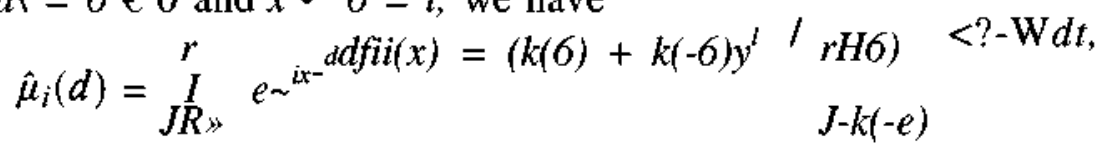


as in the proof of Lemma 3, since $e^{\alpha^{2 x}}$ is a ridge function. Here we are assuming that $K$ contains the origin, and $k(6)$ is the support function of $K$. It follows that $j i \grave{i}(d)=$ piiid) for each $d$ on a line through the origin parallel to some 6 e 0 . It is known (see [5, p. 272]) that $p, j$ is analytic, so by our assumptions $f i-f i j$ is identically zero. Since a measure is uniquely determined by its Fourier transform [5, Theorem 8.2.4], the result follows.

REMARK. In $\mathrm{R}^{2}$, any infinite set of directions satisfies the conditions of Theorem 2. In $\mathrm{R}^{3}$ more is required, as easy examples show; however, a set of directions which is infinite in each of an infinite set of planes would do.

The following result was first proved in [23], in the language of probability theory.

THEOREM 3. There is a relative width megsure $v_{n \text { in }}$ the unit ball $B_{\text {if }}^{n}$ in $\mathrm{R}$ " for the unit sphere, $S^{\prime \prime} \sim$, the set of all directions, if and only if $n=2$ or 3 .

Proof. Let $d € \mathrm{R}^{\prime \prime}, d \wedge \Lambda=6$ and $x \cdot 6=t$. Then the Fourier transform $\hat{v}_{n}$ of $v_{n}$ must satisfy

$$
\begin{aligned}
& \hat{v}_{n}(d)=f \quad e^{i x d d v_{n}(x)=\backslash I^{1} e^{-i|d| t}} \\
& \begin{array}{ll}
\mathrm{f}(\sin |d|) /|d| & (|d| \neq 0), \\
11 & (|d|=0) .
\end{array}
\end{aligned}
$$

For $n=2$, this is the Fourier trand form of the function

$$
g(x)=\left\{\begin{array}{l}
\left(1-\mathrm{i}^{*} \mathrm{i}^{2>}\right. \\
0
\end{array}\right.
$$

( WSi).

so that $v i$ is the measure with this density function (and is therefore absolutely continuous with respect to Lebesgue measure in $\mathrm{R}^{2}$ ).

For $n=3$, it is known (see, for example, [11, p. 199]) that $\mathfrak{£ 3}$ is the Fourier transform of the distribution $d(\lambda x-l) / 4 n$, so that $z B$ is normalized surface area measure in $2 ?^{3}$.

If $n>3$, the Fourier transform of $\hat{v}_{n}$ is known, since it appears in the solution of the wave equation [11, pp. 197-9], and is a distribution of higher order and not a measure. However, a more straightforward way to see that no measure exists for $n>3$ is as follows. Suppose $v_{n}$ to exist, and let $/ /$ be the projection of $v_{n}$ onto any 3-dimensional 
coordinate plane $L$, so that for Borel sets $B$ in $L$, we havf $f i(B)$ $u_{n}\left(B \times \mathrm{R}^{\prime \prime} \sim^{3)}\right.$. Then $f i$ is a relative width measure in $B$ that $j x=V T$, by Theorem 2 . By the above, the supportof $U T$ ichiesing $S^{2}$, so the support of $v_{n}$ is contained in $\left(S^{2} \times \mathrm{R}^{\prime \prime} \sim\right.$ $\mathrm{R}^{3} \mathrm{X}\{0\}$, and hence in a coordinate hyperplane. This is impossible, since $v_{n}$ must vanish on each hyperplane.

The above theorem uses ideas of K. Falconer [8, Theorem 3] for $n=$ 2 and 3 . The existence of $v_{2}$ and $V T$, have long been known. Indeed, $u_{2}$ is the projection of $(1 / 3 / 2)$, and that $1 \mathcal{B}$ has the right properties was observed by Archimedes!

THEOREM 4. Let $K$ be a convex body in R", and 0 a set ofdirections as in Theorem 2. Suppose a relative width measure $n$ in $K$ for 0 exists, the centroid of $K$ with respect to $d / x$ is at the origin, and $k(6)$ $i$ s the support function of $K$. Then $K$ contains a line segment 1 , or $a$ 2-dimensional ellipse $E$ or a 3-dimensional ellipsoid $E$, such that $k$ (6) agrees with the support function of 1 or $E$, respectively, for $0 e 0$, and ix is either

(i) normalized linear Lebesgue measure in 1 , or

(ii) the canonical relative width measure in $E$ obtained by a suitable affine transformation of $v_{2}$ or $v^{\wedge}$, respectively.

Proof. Lemma 3 shows that $k^{2(d)}=p(d)$ for 6 e 0 , where $p(6)$ is a second-degree homogeneous polynomial in 6 . It follows that $k(6)$ agrees with the support function of an m-dimensional ellipsoid for $6 € 0$, for some $m$ with $1 \leq m \leq n$. (For the non-degenerate case $m=n$, the proof is given in $[19$, p. 825 , second paragraph]. In the degenerate case, suppose $p(8)$ contains only the variables 9 , $O_{m}$ for $m<n$. Then there is an m-dimensional ellipsoid $E$ in $R^{m}$ whose support function $h(6)$ satisfies $h^{2(6)}=p(9)$. Now note that the support function of $E$ regarded as a subset of R" is still $h(6)$.) By taking an affine transformation we may assume that this ellipsoid is $B^{m .}$ Therefore $f i$ has the relative width property in $B^{m}$ for 0 , and even though the support of $n$ may a priori not lie inside $B^{m}$, we may use Theorems 2 and 3 to see that $\mathrm{m}_{1} \leq 3$ and $/ n$ is an affine image of $y_{2}$ in $B^{2}$ or $D 3$ in $B^{3}$; except in the degenerate case $m=1$, when $k(6)$ agrees with the support function of a line segment for 6 e 0 and $f i$ must be normalized linear Lebesgue measure on this segment (this is the case $n=1$ in Theorem 3). 
Since the support of $/ /$ must lie inside $K$, we see that $K$ contains the line segment, ellipse or ellipsoid, completing the proof.

Theorem 4 goes quite far in characterizing relative width measures for infinite 0 . Although constraints are also placed on $K$, it need not actually be an ellipsoid. To see this, let 0 be an infinite set of directions in $\mathrm{R}^{2}$ which is sparse enough to allow the existence of a convex body $K$, containing $B^{2}$ but different from $B^{2}$, such that for $6 e 0, K$ and $B^{2}$ have common supporting lines parallel to 6 .

4. Uniqueness for finite 0 . The results of $\S 3$ raise the question whether certain finite sets of directions might force uniqueness of the corresponding relative width measures. The next theorem uses a result from the theory of Radon transforms to show that this is not so, at least in dimensions two and three.

THEOREM 5. Let $n=2$ or 3 , and let 0 be a finite set ofdirections in $\mathrm{R}$ ". Then there are two different relative width measures in $B^{n}$ for 0 .

Proof. Let $n \geq 2$. Denote by $H(t, 6)$ the hyperplane in R" orthogonal to 6 at distance $\mathrm{V}$ from the origin. By [13, Proposition 7.6], if $K$ is any compact set there is a function / supported in $K$, infinitely differentiate on $K$ and not identically zero, such that

$$
\int_{>H(t, 0)} f(x) d m(x)=O
$$

for all / and 8 e 0 , where $m$ is ( $n$ - 1)-dimensional Lebesgue measure in the hyperplane $H(t, 8)$.

To deal with the case $n=2$, take $K=B^{2}$ and let $/$ be any such function; then / is bounded, $|/(\mathrm{x})|<M$ say. Let $g(x)$ be the density function for the measure $y_{2}$ (see Theorem 3 ) and note that $g(x)>$ $(l / 2 n)$ for $\mathrm{JC} e$ int $B^{2 .}$

$$
H(E)=f(g(x)-(f(x) / 2 n M)) d x
$$

for Borel sets $E$ in $B^{2,}$ then $V$ is a Borel measure in $B^{2}$ which is different from $v_{2}$.

Let $6 \mathrm{G} 0$, and let $S$ be the slab orthogonal to 8 bounded by the hyperplanes $H(t, d)$ and $H\left(t_{2}, 8\right)$. Then

$$
\begin{aligned}
\mu(S) & =\int_{S} g(x) d x-\frac{1}{2 \pi M} \int_{S} f(x) d x \\
& =u_{2}(S)-\hat{n}_{\bar{M}} f_{t i} I_{J_{H(t: d)}} f(x) d m(x) d t=u_{2}(S),
\end{aligned}
$$

so that $n$ is a relative width measure in $B^{2}$ for 0 , as required. 


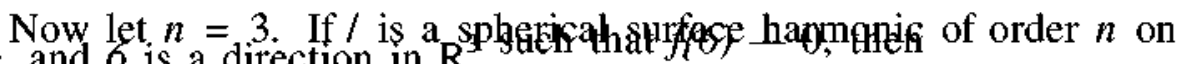
$S^{2}$, and $\sigma$ is a direction in $\mathrm{R}^{3,}$

$$
\int_{J s^{2 n H(t, e)}} f d \lambda_{t}=0
$$

where $X_{t}$ is normalized linear measure in the circle $S^{2} \mathrm{CVH}(t, 6)$. (This follows from [25, p. 100], which uses the expansion of / involving Legendre polynomials.) Suppose that $0=\left\{0, \ldots, 0^{\wedge}\right\}$ is a finite set of directions. By $[21, \S 18]$, the vector space of (pure) harmonics on $S^{2}$ of order $n$ has dimension $2 n+l$. Therefore if $n+1>k$, by linear algebra there is a spherical harmonic / of order $n$ such that $b($ (n)
for $i=1, \ldots, k$. If $\mathrm{I} / / \leq M$ on $S^{2}$ and $E$ is a Borel set in 2 ?

$$
H(E)=\sum_{E \cap S^{2}}(l-f(x) / M) d v_{3}(x) .
$$

The proof that $f i$ is a relative width measure in $\mathrm{i} ?^{3}$ for $\mathrm{O}$ which is different from 13 now follows that from the case $n=2$ above.

It remains open whether there is a relative width measure in $B^{n}$ $n \geq 4$, for the coordinate directions (or any finite set of directions).

\section{REFERENCES}

[1] R. Alexander. A problem about lines and ovals, Amer. Math. Monthly. 75 (1968). 482-487.

[2] Th. Bang, On covering by parallel-strips, Mat. Tidsskr. B, (1950), 49-53.

[3]__ A solution of the "plank problem", Proc. Amer. Math. Soc, 2 (1951), 990-993.

[4]_ Some remarks on the union of convex bodies, Tolfte Skand. Kongr., 1953, (1954), 5-11.

[5] H. Bauer, Probability Theory and Elements ofMeasure Theory, Academic Press. London (1981).

[6] M. Bognăr. On W. Fenchel's solution of the plank problem, Acta. Math. Acad. Sci. Hungar., 12 (1961), 269-270.

[7] H. G. Eggleston, On triangles circumscribing plane convex sets, J. London Math. Soc, 28(1953), 36-46.

[8] K. J. Falconer, The determination of afunction from its projections, Math. Proc. Cambridge Phil. Soc, 85 (1979), 351-355.

[9]_ Function space topologies defined by sectional integrals and applications to an extremal problem, Math. Proc Cambridge Phil. Soc, 87 (1980), 81-96.

[10] W. Fenchel. On Th. Bang's solution of the plank problem, Mat. Tidsskr. B. (1951), 49-51.

[11] I. M. Gel'fand and G. E. Shilov, Generalized Functions, Vol. I, Academic Press. New York (1964). 
[12] J. W. Green. On the determination of afunction in the plane by its integrals over straight lines, Proc. Amer. Math. Soc, 9 (1958), 758-762.

[13] S. Helgason, The Radon Transform, Birkhäuser, Boston (1980).

[14] T.-Y. Lee, J.-S. Lin, K.-C. Tong and M.-Y. Zhang, A solution ofBang's "plank problem", (in Chinese). J. Chinese Math. Soc, 2 (1953), 139-143.

[15] W. O. J. Moser, On the relative widths of coverings by convex bodies, Canad. Math. Bull., 1 (1958), 154.

[16] Y. N. Moschovakis, Descriptive Set Theory, North-Holland, Amsterdam (1980).

[17] D. Ohmann, Eine Abschà'tzung für die Dicke bei Überdeckung durch konvexe Körper, J. Reine Angew. Math., 190 (1952), 125-128.

[18]___ Kurzer Beweis einer Abschátzungfür die Breite bei Überdeckung durch konvexe Korper, Arch. Math.. 8 (1957). 150-152. (See the review in Zentralblatt für Math., 81 (1959), 165.)

[19] C. M. Petty, Surface area of a convex body under affine transformations, Proc. Amer. Math. Soc, 12 (1961), 824-828.

[20] A. Renyi, On projections of probability distributions, Acta Math. Acad. Sci. Hungar. 3 (1952), 131-142.

[21] G. Sansone, Orthogonal Functions, Robert E. Kreiger Pub. Co., New York. 1977.

[22] L. Schwartz, Radon Measures on Arbitrary Topological Spaces and Cylindrical Measures, Oxford Univ. Press, Bombay (1973).

[23] Gideon Schwarz. Multivariate distributions with uniformly distributed projections, Annals of Probab., 13 (1985), 1371-1372.

[24] A. Tarski, Further remarks about the degree of equivalence of polygons, (in Polish), Odbitka Z. Parametru., 2 (1932), 310-314.

[25] P. Ungar, Freak theorem aboutfunctions on a sphere, J. London Math. Soc, 29 (1954), 100-103.

Received June 8, 1987 and in revised form October 25, 1987. Work done while visiting UC Davis, Davis, CA 95616, USA.

DEPARTMENT OF MATHEMATICAL SCIENCES

KING FAHD UNIVERSITY OF PETROLEUM \& MINERALS

DHAHRAN 31261. SAUDI ARABIA 


\section{PACIFIC JOURNAL OF MATHEMATICS \\ EDITORS}

\author{
V. S. VARADARAJAN \\ (Managing Editor) \\ University of California \\ Los Angeles, CA 90024 \\ HERBERT CLEMENS \\ University of Utah \\ Salt Lake City, UT 84112 \\ R. FINN \\ Stanford University \\ Stanford, CA 94305
}

\author{
HERMANN FLASCHKA \\ University of Arizona \\ Tucson, AZ 85721
}

vAUGHAN F. R. JONES

University of California

Berkeley, CA 94720

STEVENKERCKH OFF

Stanford University

Stanford, CA 94305

\author{
ROBION KIRBY \\ University of California \\ Berkeley, CA 94720 \\ C. C. MOORE \\ University of California \\ Berkeley, CA 94720 \\ HAROLD T ARK
}

University of California, San Diego La Jolla, CA 92093

\section{ASSOCIATE EDITORS}
R. ARENS
E. F. BECKENBACH
B. H. NEUMANN
F. WOLF
K. YOSHIDA

(1906-1982)

\section{SUPPORTING INSTITUTIONS}

\author{
UNIVERSITY OF ARIZONA \\ UNIVERSITY OF BRITISH COLUMBIA \\ CALIFORNIA INSTITUTE OF TECHNOLOGY \\ UNIVERSITY OF CALIFORNIA \\ MONTANA STATE UNIVERSITY \\ UNIVERSITY OF NEVADA, RENO \\ NEW MEXICO STATE UNIVERSITY \\ OREGON STATE UNIVERSITY
}

\author{
UNIVERSITY OF OREGON \\ UNIVERSITY OF SOUTHERN CALIFORNIA \\ STANFORD UNIVERSITY \\ UNIVERSITY OF HAWAII \\ UNIVERSITY OF TOKYO \\ UNIVERSITY OF UTAH \\ WASHINGTON STATE UNIVERSITY \\ UNIVERSITY OF WASHINGTON
}

The Supporting Institutions listed above contribute to the cost of publication of this Journal, but they are not owners or publishers and have no responsibility for its content or policies.

Mathematical papers intended for publication in the Pacific Journal of Mathematics should be in typed form or offset-reproduced (not dittoed), double spaced with large margins. Please do not use built up fractions in the text of the manuscript. However, you may use them in the displayed equations. Underline Greek letters in red, German in green, and script in blue. The first paragraph must be capable of being used separately as a synopsis of the entire paper. In particular it should contain no bibliographic references. Please propose a heading for the odd numbered pages of less than 35 characters. Manuscripts, in triplicate, may be sent to any one of the editors. Please classify according to the scheme of Math. Reviews, Index to Vol. 39. Supply name and address of author to whom proofs should be sent. All other communications should be addressed to the managing editor, or Elaine Barth, University of California, Los Angeles, California 90024.

There are page-charges associated with articles appearing in the Pacific Journal of Mathematics. These charges are expected to be paid by the author's University, Government Agency or Company. If the author or authors do not have access to such Institutional support these charges are waived. Single authors will receive 50 free reprints; joint authors will receive a total of 100 free reprints. Additional copies may be obtained at cost in multiples of 50 .

The Pacific Journal of Mathematics is issued monthly as of January 1966. Regular subscription rate: $\$ 190.00$ a year (5 Vols., 10 issues). Special rate: $\$ 95.00$ a year to individual members of supporting institutions.

Subscriptions, orders for numbers issued in the last three calendar years, and changes of address should be sent to Pacific Journal of Mathematics, P.O. Box 969, Carmel Valley, CA 93924, U.S.A. Old back numbers obtainable from Kraus Periodicals Co., Route 100, Millwood, NY 10546.

The Pacific Journal of Mathematics at P.O. Box 969, Carmel Valley, CA 93924 (ISSN 0030-8730) publishes 5 volumes per year. Application to mail at Second-class postage rates is pending at Carmel Valley, California, and additional mailing offices. Postmaster: send address changes to Pacific Journal of Mathematics, P.O. Box 969, Carmel Valley, CA 93924.

\section{PUBLISHED BY PACIFIC JOURNAL OF MATHEMATICS, A NON-PROFIT CORPORATION} Copyright (C) 1988 by Pacific Journal of Mathematics 


\section{Pacific Journal of Mathematics}

Vol. 135, No. $2 \quad$ October, 1988

Waleed A. Al-Salam and Mourad Ismail, $q$-beta integrals and the

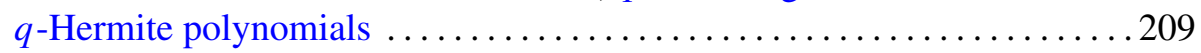

Johnny E. Brown, On the Ilieff-Sendov conjecture $\ldots \ldots \ldots \ldots \ldots \ldots \ldots 223$

Lawrence Jay Corwin and Frederick Paul Greenleaf, Spectrum and

multiplicities for restrictions of unitary representations in nilpotent Lie

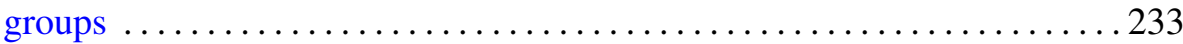

Robert Jay Daverman, 1-dimensional phenomena in cell-like mappings on 3-manifolds .......................................... 269

P. D. T. A. Elliott, A localized Erdős-Wintner theorem .............. 287

Richard John Gardner, Relative width measures and the plank problem . . . 299

F. Garibay, Peter Abraham Greenberg, L. Reséndis and Juan José

Rivaud, The geometry of sum-preserving permutations ............313

Shanyu Ji, Uniqueness problem without multiplicities in value distribution

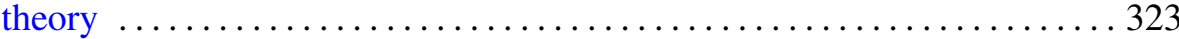

Igal Megory-Cohen, Finite-dimensional representation of classical

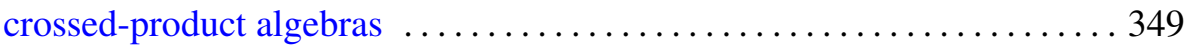

Mirko Navara, Pavel Pták and Vladimír Rogalewicz, Enlargements of

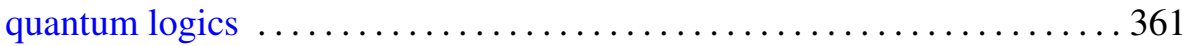

Claudio Nebbia, Amenability and Kunze-Stein property for groups acting

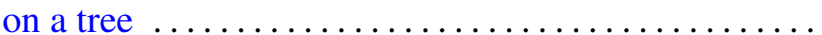

Chull Park and David Lee Skoug, A simple formula for conditional Wiener

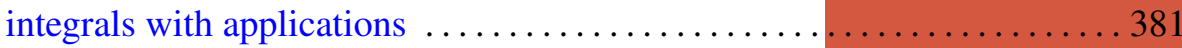

Ronald Scott Irving and Brad Shelton, Correction to: "Loewy series and simple projective modules in the category $\mathrm{O}_{s} " \ldots .$.

Robert Tijdeman and Lian Xiang Wang, Correction to: "Sums of products

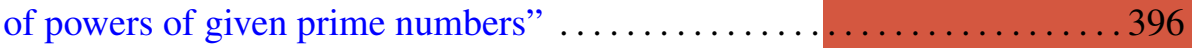

